\title{
Pathologic Caveolin-1 Regulation of PTEN in Idiopathic Pulmonary Fibrosis
}

\author{
Hong Xia, ${ }^{*}$ Wajahat Khalil, ${ }^{*}$ Judy Kahm, ${ }^{*}$ \\ Jose Jessurun, ${ }^{\dagger}$ Jill Kleidon, ${ }^{*}$ and Craig A. Henke* \\ From the Departments of Medicine, " and Laboratory Medicine \\ and Pathology, University of Minnesota, Minneapolis, Minnesota
}

Idiopathic pulmonary fibrosis (IPF) is a progressive fibroproliferative disorder refractory to current pharmacological therapies. Fibroblasts isolated from IPF patients display pathological activation of PI3K/Akt caused by low PTEN phosphatase activity. This enables these cells to escape the negative proliferative properties of polymerized collagen. The mechanism underlying low PTEN activity in IPF fibroblasts is unclear, but our prior studies indicate that membrane-associated PTEN expression is decreased in these cells. Caveolin-1 is an integral membrane protein whose expression is decreased in IPF lung tissue, but how low caveolin-1 contributes to pathological fibrosis is incompletely understood. The objective of this study was to examine the hypothesis that caveolin-1 regulates PTEN function in IPF fibroblasts. Here we demonstrate that caveolin-1 expression is a determinant of membrane PTEN levels and show that PTEN interacts with caveolin-1 via its caveolin-1-binding sequence. We demonstrate that caveolin-1 expression is low in IPF fibroblasts and that this correlates with low membrane PTEN levels, whereas overexpression of caveolin-1 restores membrane PTEN levels, inhibits Akt phosphorylation, and suppresses proliferation. We demonstrate that caveolin-1 and PTEN expression are low in myofibroblasts within IPF fibroblastic foci. These data indicate that IPF fibroblasts display low caveolin-1 expression, which results in low membrane-associated PTEN expression. This creates a membrane microenvironment depleted of inhibitory phosphatase activity, facilitating the aberrant activation PI3K/Akt and pathological proliferation. (Am J Pathol 2010, 176:2626-2637; DOI: 10.2353/ajpath.2010.091117)

Polymerized type I collagen suppresses normal fibroblast proliferation. ${ }^{1-4}$ The mechanism involves inhibition of the PI3K/Akt signal by high PTEN phosphatase activity. ${ }^{1}$ This provides an effective physiological mechanism to limit fibroproliferation after tissue injury. In contrast, inappropriately low PTEN activity has been implicated in the pathogenesis of human fibroproliferative disorders, and PTEN-deficient mice display increased lung fibrosis in response to injury attributable to a durable fibroproliferative response. ${ }^{1,5}$ Importantly, lung fibroblasts isolated from patients with idiopathic pulmonary fibrosis (IPF), a prototypical fibroproliferative disorder, display depleted membrane PTEN levels and consequently inappropriately low PTEN activity in response to their interaction with polymerized collagen via $\beta 1$ integrin. ${ }^{1}$ This results in pathological activation of the integrin/PI3K/Akt signal pathway, which enables them to elude the proliferationsuppressive effects of polymerized collagen. The molecular mechanism underlying this inappropriately low PTEN function in IPF fibroblasts remains to be elucidated.

PTEN is a dual lipid/protein phosphatase that negatively regulates proliferation by repressing the integrin-PI3K/Akt pathway. ${ }^{6-13}$ It has relatively high constitutive phosphatase activity consistent with its function as a tumor suppressor. A current paradigm for PTEN regulation suggests that the phosphorylation of key serine/threonine residues in the PTEN C-terminal tail determine protein stability and activation. Evidence suggests that PTEN activation involves dephosphorylation of serine/threonine residues within the $\mathrm{C}$ terminus and translocation from the cytosol to the plasma membrane where it is in the right location to inhibit phosphoinositol 3,4,5-triphosphate. ${ }^{14-16}$ However, the precise mechanism for PTEN localization to the plasma membrane and its activation remain incompletely understood.

Caveolin-1 is an integral membrane protein that regulates a variety of cellular processes, including integrin turnover and signal transduction pathways controlling cell proliferation and apoptosis, such as the PI3K/Akt signal pathway. ${ }^{17,18}$ Studies indicate that similar to PTEN haplo-insufficient mice, caveolin-1-deficient mice also display an increased propensity for the development of

Supported by National Heart, Lung, and Blood Institute (NHLBI) grants R01 HL074882 and P01 HL91775 (to C.A.H.).

H.X. and W.K. contributed equally to this study.

Accepted for publication February 4, 2010

Supplemental material for this article can be found on http://ajp. amjpathol.org.

Address reprint requests to Craig A. Henke, M.D., Box 276, University of Minnesota, 420 Delaware Street SE, Minneapolis, MN 55455. E-mail: henke002@umn.edu. 
lung fibrosis. ${ }^{19,20}$ Interestingly, caveolin-1 expression has been found to be low in fibrotic lung tissue from patients with IPF, but the mechanism by which caveolin-1 deficiency results in exaggerated fibrosis is still incompletely understood. ${ }^{21}$ However, amino acid sequence analysis of PTEN indicates that PTEN contains the caveolin-1 consensus binding sequence $\Phi X \Phi X X X X \Phi$ corresponding to amino acids 271-278 (FHFWVNTF), where $\Phi=$ aromatic amino acid phenylalanine $(F){ }^{22}$ This suggests a relationship between caveolin-1 expression and PTEN function.

Here we demonstrate that the level of caveolin-1 protein expression is a determinant of membrane-associated PTEN levels and activity. The mechanism involves direct interaction of caveolin-1 with PTEN via PTEN's caveolin-1-binding sequence. We demonstrate that caveolin-1 protein expression is decreased in IPF fibroblasts cultured on polymerized collagen compared with control fibroblasts, and this corresponds to low membrane PTEN expression and augmented levels of phosphorylated Akt. However, overexpression of caveolin-1 in IPF fibroblasts augments PTEN levels, reduces the level of phospho-Akt, and suppresses their ability to proliferate. We show that there is a low level of caveolin-1 and PTEN expression in myofibroblasts comprising fibroblastic foci in human IPF lung tissue and that within the fibroblastic focus, the pattern of caveolin-1 expression correlates well with that of PTEN. Our data suggest that in IPF fibroblasts, reduced caveolin-1 expression at the plasma membrane creates a membrane microenvironment depleted of PTEN phosphatase activity and favorable for the pathological activation of the PI3K/Akt signal pathway. This confers IFP fibroblasts with the ability to elude the proliferation-suppressive effects of polymerized type I collagen.

\section{Materials and Methods}

\section{Primary IPF and Normal Fibroblast Lines}

Eight primary fibroblast lines were established from IPF patients. Cells were obtained from lungs removed at the time of transplantation or death. The diagnosis of IPF was supported by history, physical examination, pulmonary function tests, and typical high-resolution chest computed tomography findings of IPF. In all cases, the diagnosis of IPF was confirmed by microscopic analysis of lung tissue and demonstrated the characteristic morphological findings of usual interstitial pneumonia. All patients fulfilled the criteria for the diagnosis of IPF as established by the American Thoracic Society and European Respiratory Society. ${ }^{23}$ Six nonfibrotic primary control adult human lung fibroblast lines were used. These lines were established from normal lung tissue $(n=3)$, or histologically normal lung tissue adjacent to carcinoid tumor $(n=2)$ or adjacent to radiationinduced fibrotic lung tissue $(n=1)$. Primary lung fibroblast lines were generated by explant culture and cultured in high-glucose DMEM containing 10\% FCS. Fibroblasts were used between passages 5 and 8. Cells were characterized as fibroblasts as described. ${ }^{24}$ Use of human tissues was approved by the Institutional Review Board at the University of Minnesota.

\section{Cell Culture}

Primary lung fibroblasts (ATCC) were cultured in high glucose DMEM containing 10\% fetal calf serum. Primary mouse lung fibroblasts, isolated from wild-type caveolin-1 (cav-1 wild-type) or caveolin-1 knock-out mice (cav-1 KO), were a gift from Augustine Choi (Harvard University, Cambridge, MA). PTEN wild-type and null fibroblasts were obtained from Eric White (University of Michigan, Ann Arbor, MI). The cells were cultured in high-glucose DMEM containing 10\% fetal calf serum. The fibroblasts were used between passages 5 and 8 for all experiments.

\section{Antibodies and Chemicals}

Anti-Cyclin D1, GAPDH, $\alpha$-SMA antibodies were obtained from Santa Cruz Biotechnology Company (Santa Cruz, CA). Caveolin-1, keratin 8/18, PTEN, HA, and phosphoAkt (Ser473) antibodies were obtained from Cell Signaling Technology (Danvers, MA).

\section{Collagen Matrices}

Three-dimensional polymerized collagen matrices (final concentration, $2 \mathrm{mg} / \mathrm{ml}$ ) were prepared by neutralizing the collagen solution with $1 / 6$ volume of $6 \times$ DMEM and diluting to a final volume with $1 \times$ DMEM to which fetal calf serum was added at a final concentration of $1 \%$ fetal calf serum. Gels formed after incubation of this solution at $37^{\circ} \mathrm{C}$ for 1 to 2 hours as described previously. ${ }^{9-11}$

\section{Adenoviral Vectors}

Adenoviral vectors containing wild-type PTEN(Ad-wtPTEN), mutant PTEN(ad-mPTEN), and control (Ad-GFP) constructs were purified according to the manufacturer's instructions (Takara Shuzo Co, Ltd., Kyoto, Japan). The adenoviral vector containing wild-type caveolin-1 was a gift from Augustine Choi (Harvard University). The cells were infected with adenoviral vectors at a multiplicity of infection of 1:20.

\section{Caveolin-1 siRNA and Control siRNA}

Caveolin-1 and control siRNA were obtained from Invitrogen. Caveolin-1 siRNA is from Validated Stealth RNAi duoPak. Control siRNA is from Stealth RNA interference negative control duplexes. Transient transfection of normal lung fibroblasts was performed using FuGENE. HD siRNA transfection reagent was obtained from Roche Applied Science (Indianapolis, IN) and used according to the manufacturer's instructions.

\section{Apoptosis Assay}

Quantification of apoptosis was performed by DNA content assay and fluorescent-based assay for detection of active caspases in cells undergoing apoptosis (CHEMICON International, Inc., Billerica, MA). Briefly, the cells were fixed in 
ice-cold $70 \%$ alcohol overnight and stained with $2 \mu \mathrm{g} / \mathrm{ml}$ propidium iodide, $100 \mu \mathrm{g} / \mathrm{ml}$ DNase in $1 \times$ phosphatebuffered saline buffer for 60 minutes. The percentage of cells in each phase of the cell cycle was quantified by FACS analysis using FACSCalibur software.

\section{Western Blot and Immunoprecipitation}

Serum-starved fibroblasts were plated on extracellular matrix-coated plates and lysed at the indicated times using cell lysis buffer containing $150 \mathrm{mmol} / \mathrm{L} \mathrm{NaCl}, 1$ mmol/L EGTA, 50 mmol/L Tris, pH 7.4, 1\% Triton X-100, $1 \%$ Nonidet P-40, $1 \%$ sodium deoxycholate (SDS), with protease inhibitors (complete protease inhibitor mixture tablets; Roche Applied Science). To isolate the cell membrane fraction, cells were first lysed with $25 \mathrm{mmol} / \mathrm{L}$ Tris$\mathrm{HCl}, \mathrm{pH} 7.4,50 \mathrm{mmol} / \mathrm{L} \mathrm{NaCl}, 1 \mathrm{mmol} / \mathrm{L}$ EDTA, 0.5\% Triton X-100, followed by centrifugation, and the pellets containing the membrane fraction lysed in lysis buffer containing Triton X-100, Nonidet P-40, and SDS (see above). Western analysis was performed on the resulting lysates. For immunoprecipitation assay, the cells were lysed in lysis buffer containing $20 \mathrm{mmol} / \mathrm{L}$ Tris- $\mathrm{HCl}, \mathrm{pH}$ 7.5, $150 \mathrm{mmol} / \mathrm{L} \mathrm{NaCl}, 1 \%$ Nonidet P-40, 1 mmol/L EDTA, $1 \mathrm{mmol} / \mathrm{L}$ EGTA, $1 \mathrm{mmol} / \mathrm{L}$ phenylmethylsulfonyl fluoride, $1 \mathrm{mg} / \mathrm{ml}$ aprotinin, protease, and phosphatase inhibitor cocktails. The samples were centrifuged at $20,000 \mathrm{~g}$ for 15 minutes at $4^{\circ} \mathrm{C}$, and the lysates were precleared for 1 hour at room temperature with protein $A / G$ beads and immunoprecipitated for 16 hours at $4^{\circ} \mathrm{C}$ with the appropriate primary antibody. The samples were processed for Western analysis.

\section{Cell-Free in Vitro Pull-Down Assay}

Recombinant caveolin-GST protein was obtained from Abnova (Taipei, Taiwan). Recombinant PTEN-His protein was from R\&D Systems (Minneapolis, MN). $0.5 \mu \mathrm{g}$ of recombinant caveolin-1 and recombinant PTEN protein, either alone or mixed together, were added to the reaction buffer $(120 \mathrm{mmol} / \mathrm{L} \mathrm{NaCl}, 10 \mathrm{mmol} / \mathrm{L} \mathrm{KCl}, 2 \mathrm{mmol} / \mathrm{L}$ $\mathrm{KPO} 4, \mathrm{pH} 7.4$ in PBS) containing GST-beads and incubated $\left(4^{\circ} \mathrm{C}, 16\right.$ hours). After centrifugation, the beads were washed $3 \times$ with wash buffer $(50 \mathrm{mmol} / \mathrm{L}$ Tris, $\mathrm{pH}$ $7.4,150 \mathrm{mmol} / \mathrm{L} \mathrm{NaCl}, 0.02 \%$ triton X-100). Western analysis was then performed.

\section{Proliferation Assay}

Fibroblasts were serum-starved for 2 days and then plated on monomeric $(100 \mu \mathrm{g} / \mathrm{ml})$ or on top of polymerized collagen $(2 \mathrm{mg} / \mathrm{ml})$ matrices in DMEM $+1 \%$ fetal calf serum. After the cells were replated onto polymerized collagen matrices, the media were replaced with DMEM + $10 \%$ FBS. The cells were incubated with $10 \mu \mathrm{mol} / \mathrm{L} \mathrm{BrdU}$ for 5 hours before the cells were harvested at 24 hours. The cells were then stained with anti-BrdU antibody to quantify DNA synthesis and 7-amino actinomycin D as a measure of total DNA. DNA synthesis was quantified by assessing the percentage of BrdU-positive cells by FACS according to the manufacturer's instructions (BD Biosciences, San Diego, CA).

\section{In Vitro Phosphatase Assay}

PTEN was immunoprecipitated from cell lysates with $4 \mu \mathrm{g}$ of anti-PTEN antibody. $10 \mu \mathrm{l}$ of PTEN enzyme assay buffer (100 mmol/L Tris-HCl, $\mathrm{pH} 8.0$, and $2 \mathrm{mmol} / \mathrm{L}$ dithiothreitol) and $10 \mu \mathrm{l}$ of phospholipid vesicles (PLV; 0.1 $\mathrm{mmol} / \mathrm{L} \mathrm{diC_{8 }} \mathrm{PIP}_{3}, 0.5 \mathrm{mmol} / \mathrm{L}$ dioleoyl phosphatidylserine

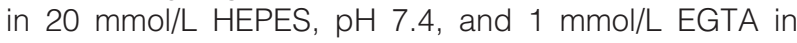
$\mathrm{diC}_{8} \mathrm{PIP}_{3}$ followed by sonication for 30 minutes) were added to $5 \mu \mathrm{l}$ of immunoprecipitate and incubated. The enzyme reaction was terminated by adding $100 \mu \mathrm{l}$ of Malachite Green solution. PTEN activity was measured using a microtiter plate reader at $630 \mathrm{~nm}$.

\section{Immunofluorescent Confocal Microscopy}

Immunostaining was performed on paraffin-embedded IPF and control lung tissue using a Zeiss Axiovert 200M Confocal Microscope.

\section{Immunofluorescence}

Immunofluorescence studies were performed on caveolin-1-null and wild-type fibroblasts cultured on glass coverslips. The cells were plated on glass coverslips and infected with the adenoviral vector containing wild-type caveolin-1 construct for 24 hours. The cells were then fixed, permeabilized, and incubated (60 minutes, room temperature) with the appropriate primary antibody (PTEN or Cav1). The cells were then incubated (60 minutes, room temperature) with cy2- or cy3-conjugated secondary antibodies, followed by incubation (10 minutes, room temperature) with DAPI. Primary antibodies caveolin-1 and total PTEN (6H2.1) were obtained from Cell Signaling and Cascade Bioscience (Winchester, MA), respectively.

\section{Data Analysis}

Comparisons of data among each experiment were performed with the unipolar unpaired or paired Student $t$ test. All experiments were replicated a minimum of three times. Data are expressed as mean \pm SD. $P<0.05$ were considered significant.

\section{Results}

\section{Myofibroblasts within IPF Fibroblastic Foci Display Low-Intensity Expression of Both Caveolin-1 and PTEN Protein}

The sentinel morphological lesion of IPF is the fibroblastic focus. Fibroblastic foci consist of a subepithelial accumulation of $\alpha$ smooth muscle actin expressing fibroblasts in a type I collagen-rich matrix. We have found that membrane PTEN levels are decreased in primary fibro- 
A



Figure 1. Myofibroblasts within IPF fibroblastic foci display low-intensity expression of both caveolin-1 and PTEN protein. H\&E and immunofluorescent confocal microscopic analysis were performed on control human lung tissue $(n=4)$ and IPF tissue $(n=3)$. Shown is representative H\&E staining of a fibroblastic foci (FF) in IPF tissue (A). Immunofluorescent confocal microscopic analysis was performed on the IPF fibroblastic foci within the boxed region shown in $\mathbf{A}$ and illustrates the pattern of caveolin-1 (Cav-1), PTEN, $\alpha$-smooth muscle actin ( $\alpha$-SMA), and keratin $8 / 18$ expression $(\mathbf{B}-\mathbf{K})$. Insets in $\mathbf{B}$ and $\mathbf{F}$ display the pattern of caveolin-1 and PTEN staining in myofibroblasts within the fibroblastic focus in a zone beneath the epithelium and in the overlying epithelium, respectively. Myofibroblasts are identified by $\alpha$-smooth muscle actin staining, epithelial cells by keratin staining, and nuclei are identified by DAPI staining. Please note that "Merge" indicates merging of caveolin-1 (red) with $\alpha$-SMA (green) or PTEN (red) with $\alpha$-SMA (green). $\mathbf{L}-\mathbf{N}$ Controls performed on IPF lung tissue consisting of secondary antibody without primary (Ctrl-Cy3, Ctrl-Cy2). O-R: Immunostaining of control lung tissue demonstrating prominent caveolin-1 and PTEN staining in cells lining the alveolar airspace. Scale bar $=50 \mu \mathrm{m}$.
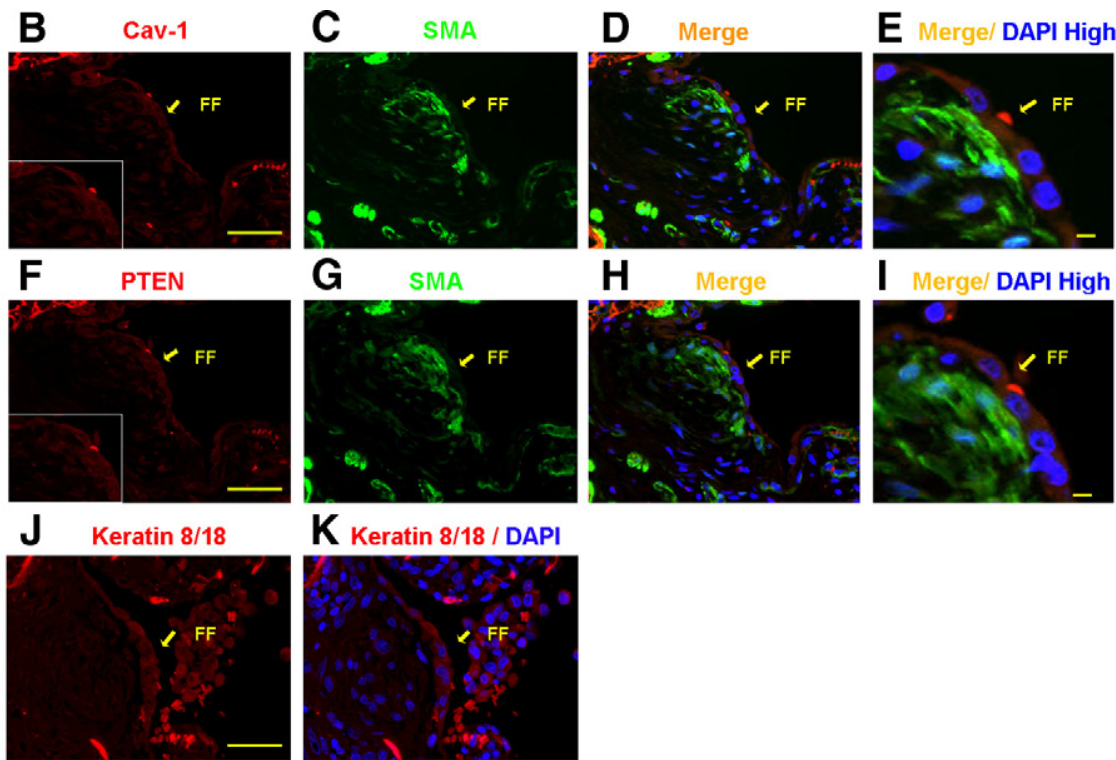

K Keratin 8/18 / DAPI
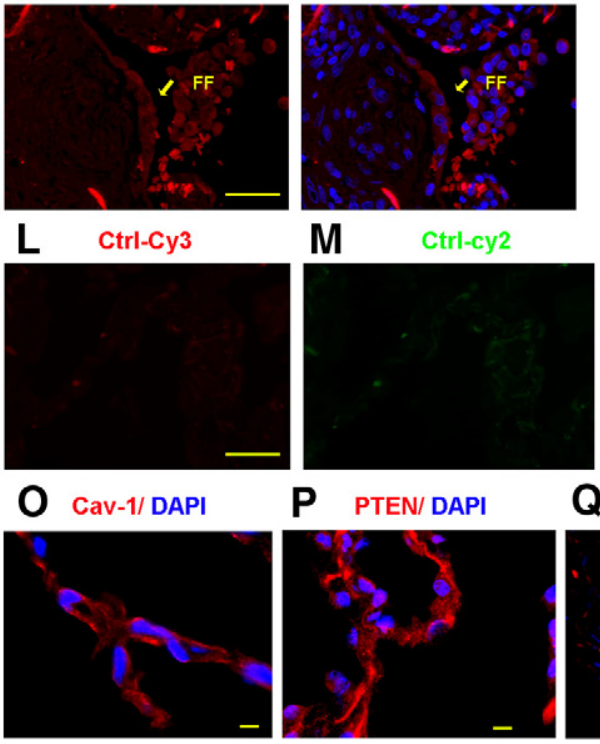
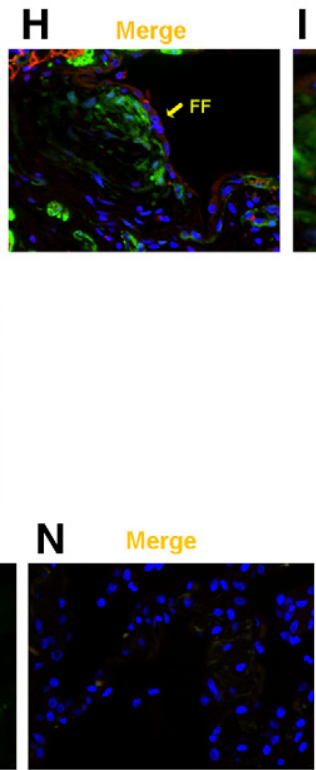

I Merge/ DAPI High
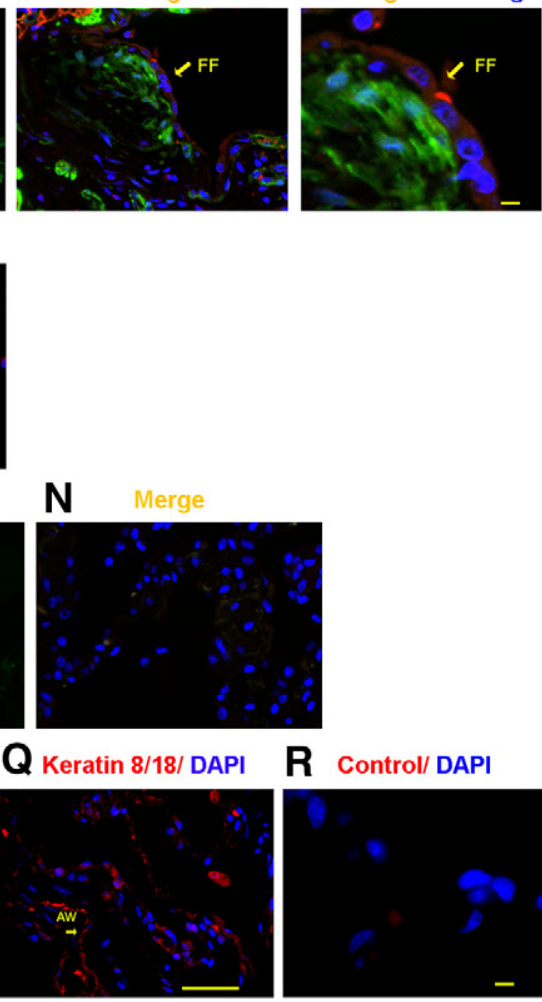

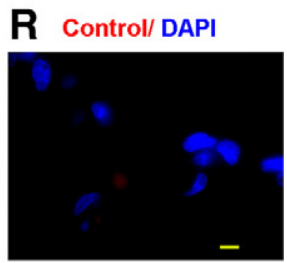

blasts derived from patients with IPF, and a previous study has found that caveolin-1 expression is decreased in IPF lung tissue and fibroblasts. ${ }^{1,21}$ Together, these data suggest a potential relationship between caveolin-1 and PTEN expression in IPF. To begin to address this issue, we analyzed the pattern of caveolin-1 and PTEN expression within IPF fibroblastic foci by immunofluorescence confocal microscopy and compared it to human control lung tissue (Figure 1A, H\&E, L-N staining of a fibroblastic focus). $\alpha$ smooth muscle actin was used as a marker of myofibroblasts and keratin 8/18 as an epithelial marker. Several differences in caveolin-1 and PTEN staining features between the IPF fibroblastic focus and control lung tissue were noteworthy. First, when analyzing IPF lung tissue, we noted that prominent caveolin-1 and PTEN expression was displayed in cells overlying the fibroblastic foci (Figure 1B for caveolin-1 and F for PTEN). These cells stained positive for the epithelial cell marker keratin $8 / 18$, suggesting that they are epithelial (Figure 1 , $\mathrm{J}$ and $\mathrm{K}$ ). $\alpha$ smooth muscle actin staining was not apparent in the epithelial cells overlying the fibroblastic foci. In contrast, cells constituting the fibroblastic foci, especially those in a zone just beneath the overlying epithelial cells, stained prominently for $\alpha$ smooth muscle actin indicating that these cells are myofibroblasts (Figure 1, C and $G$ ).
Myofibroblasts within the fibrotic foci displayed relatively low intensity expression of both caveolin-1 and PTEN compared with the overlying epithelial cells (Figure 1, merged panels $D$ and $E$ showing caveolin-1 and $\alpha$-smooth muscle actin and merged panels $\mathrm{H}$ and I showing PTEN and $\alpha$-smooth muscle actin). Second, when we analyzed control lung tissue, we found that both caveolin-1 and PTEN expression were very prominent in cells lining the alveolar airspace (Figure 1, O-R), many of which stained positive for the epithelial cell marker keratin $8 / 18$. The vast majority of caveolin-1 and PTEN staining cells lining the normal alveolar airspace did not stain for $\alpha$ smooth muscle actin (see supplemental Figure 1 at http://ajp.amjpathol.org). Rare cells within the alveolar wall stained for both vimentin and caveolin-1, suggesting that interstitial fibroblasts may stain for cavolin-1 (see supplemental Figure 2 at http://ajp.amjpathol.org). Taken together, these data demonstrate that myofibroblasts within the fibroblastic focus display relatively low intensity expression of caveolin-1 and PTEN compared with epithelial cells overlying the fibroblastic focus or lining the normal alveolar airspace. In addition, the level of caveolin-1 and PTEN expression in the myofibroblasts correlates well with one another. 
Control IPF

\section{$\begin{array}{llllllllllll}1 & 2 & 3 & 4 & 5 & 6 & 1 & 2 & 3 & 4 & 5 & 6\end{array}$}

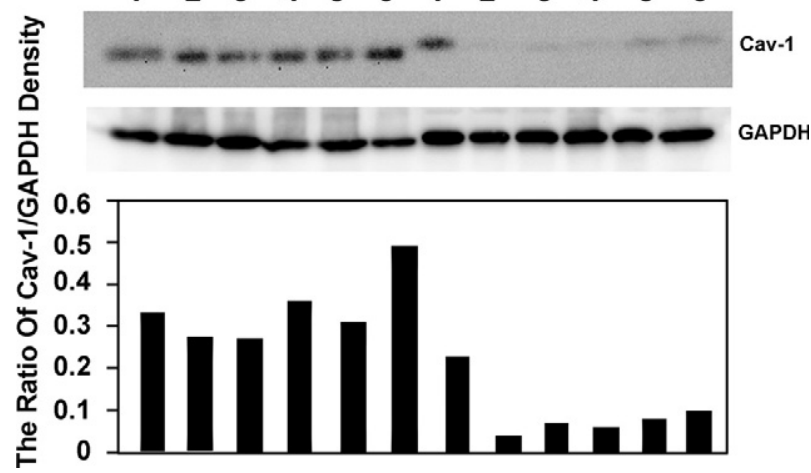

Figure 2. Caveolin-1 expression is low in IPF fibroblasts. Western analysis of caveolin-1 protein expression in primary lung fibroblasts from IPF $(n=6)$ and control patients $(n=6)$ seeded on polymerized type I collagen matrices. GAPDH is shown as a loading control. Relative protein expression was determined as the densitometric ratio of PTEN to GAPDH. Data are representative of three independent experiments.

\section{Caveolin-1 Expression Is Low in IPF Fibroblasts}

We have previously found that membrane PTEN levels are decreased in IPF fibroblasts. ${ }^{1}$ To discern whether PTEN levels correlate with caveolin-1 levels in our primary IPF fibroblast lines, we analyzed caveolin-1 expression in primary lung fibroblasts derived from IPF patients $(n=6)$ and from control patients $(n=6)$ by Western analysis. We found that caveolin-1 expression was decreased in primary IPF fibroblast cell lines compared with controls (Figure 2), consistent with a prior report. ${ }^{21}$

\section{Caveolin-1 Expression Is a Determinant of Membrane-Associated PTEN Levels}

PTEN inhibits the integrin/PI3K/Akt signal pathway. Its activation is believed to involve translocation from the cytoplasm to the membrane where it is activated and in the right location to inhibit PI3K/Akt. Because caveolin-1 is an integral membrane protein, which also regulates signal transduction including the PI3K/Akt pathway, this suggested that low caveolin-1 expression may be a determinant of low membrane PTEN levels in IPF fibroblasts. To analyze whether caveolin-1 expression regulates membrane-associated PTEN levels, we used caveolin-1 wild-type and null lung fibroblasts. We found that caveolin-1-null fibroblasts displayed low levels of membraneassociated PTEN compared with wild-type controls, and this was associated with an augmented level of phosphoAkt (Figure 3A). Consistent with this immunofluorescence analysis showed that caveolin-1-null fibroblasts displayed a faint cytoplasmic distribution of PTEN, whereas PTEN expression was more intense in wild-type cells (data not shown). We next examined the effect of reconstituting caveolin-1 into caveolin-1-null fibroblasts using an adenoviral vector containing a wild-type caveolin-1 construct on membrane PTEN levels. Caveolin-1-null cells reconstituted with caveolin-1 now displayed caveolin-1 expression. Caveolin-1-null cells transfected with an empty vector did not express caveolin-1. Caveolin-1-null

\section{A Cav-1WT Cav-1Null}

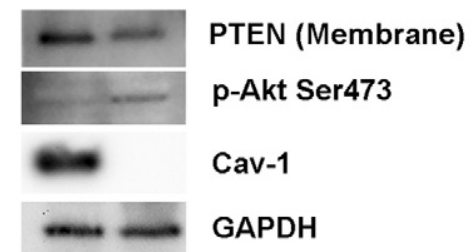

B Ad-GFP Ad-Cav-1

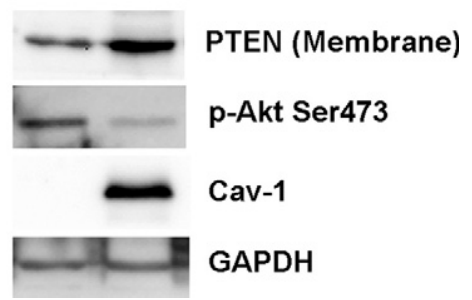

Figure 3. Caveolin-1 expression is a determinant of membrane PTEN levels. A: Caveolin-1-null and wild-type lung fibroblasts were seeded on polymerized collagen and examined for caveolin-1, membrane PTEN, and phosphoAkt expression using GAPDH as a loading control. B: Caveolin-1-null fibroblasts were reconstituted with wild-type caveolin-1 (Ad-Cav-1) and seeded on polymerized collagen. Cells infected with empty vector are shown as a control (Ad-GFP). Shown is Western analysis of caveolin-1, membrane PTEN, phospho-Akt, and GAPDH levels.

fibroblasts reconstituted with caveolin-1 displayed increased levels of membrane-associated PTEN and a lower level of phospho-Akt compared with cells transfected with empty vector (Figure 3B). These data indicate that the level of caveolin-1 expression is a determinant of the amount of PTEN associated with the membrane.

\section{PTEN Associates with Caveolin-1 in Control Fibroblasts, but an Association Cannot be Detected in IPF Fibroblasts}

Because caveolin-1 is a determinant of PTEN expression and the pattern of caveolin-1 expression correlates with PTEN expression, we next sought to assess whether PTEN associates with caveolin-1. We first analyzed whether we could detect a binding association between endogenous PTEN and caveolin-1 in control and IPF fibroblasts. An endogenous caveolin-1/PTEN complex could be detected in control fibroblasts (Figure 4A; see also Figure 5B), where endogenous caveolin-1 and membrane PTEN levels are relatively high. In contrast, both membrane PTEN and caveolin-1 levels are very low in IPF fibroblasts. Not surprisingly, we were unable to detect a binding interaction between endogenous caveolin-1 and PTEN in IPF fibroblasts (data not shown). However, when caveolin-1 was overexpressed in IPF fibroblasts, a binding interaction between caveolin-1 and endogenous PTEN could be detected (Figure 4B). These data suggest that either a caveolin-1/PTEN complex does not form in IPF fibroblasts or that because of the very low levels of endogenous caveolin-1 and membrane PTEN, a complex is below the limits of detection.

To confirm this binding association, we overexpressed caveolin-1 in caveolin-1-null fibroblasts and performed immunoprecipitation experiments. Caveolin-1-null fibro- 
A

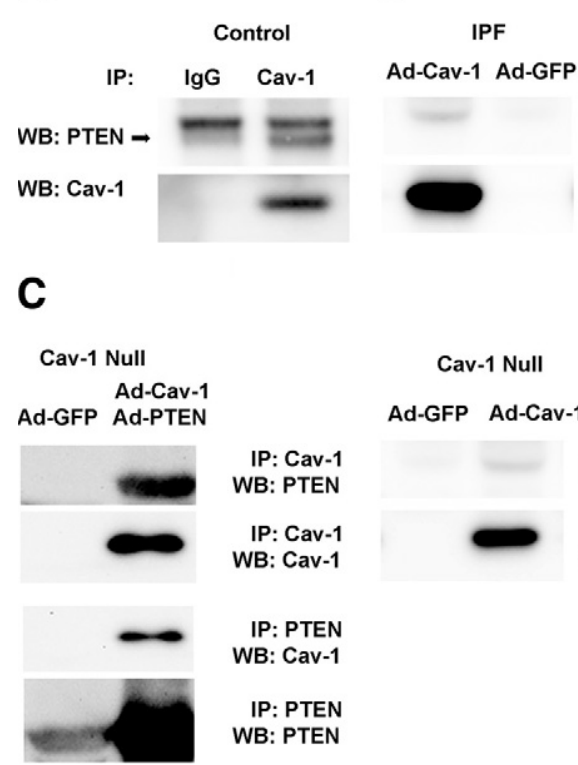

D

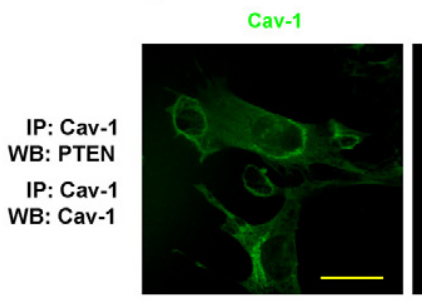

E

Ad-GFP

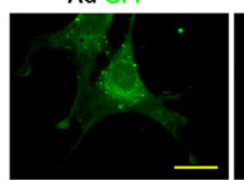

Ad-GFP

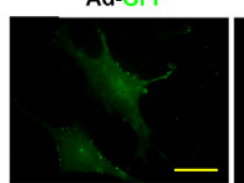

Cav-1 Null
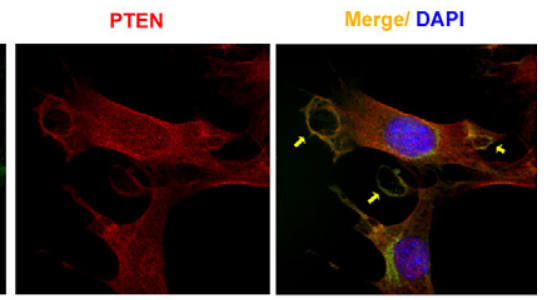

Cav-1 Null

PTEN

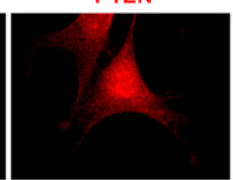

Cav-1

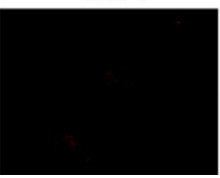

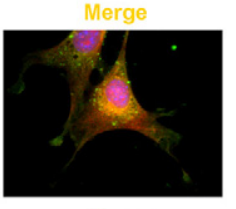

Merge

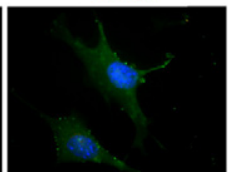

Figure 4. PTEN associates with caveolin-1 in control fibroblasts, but an association cannot be detected in IPF fibroblasts. A: Caveolin-1 was immunoprecipitated from control and IPF fibroblasts, and Western analysis for PTEN or caveolin-1 was performed (data shown for control only). B: IPF fibroblasts were infected with an adenoviral vector containing wild-type caveolin-1 construct. Cells infected with empty vector (Ad-GFP) served as a control. Caveolin-1 was immunoprecipitated, and Western analysis for PTEN and caveolin-1 was performed. C: Caveolin-1-null fibroblasts overexpressing wild-type caveolin-1 and PTEN constructs together (left) or wild-type caveolin-1 alone (right) were seeded on polymerized collagen. Caveolin-1 or PTEN was immunoprecipitated and Western analysis for PTEN, and caveolin-1 was performed. D and E: Shown is confocal microscopic immunofluorescent analysis of caveolin-1 and PTEN expression in caveolin-1-null fibroblasts reconstituted with caveolin-1 (D) or empty vector (E; GFP control) and seeded on polymerized collagen. Arrows denote colocalization of PTEN and caveolin-1 at the membrane in caveolin-1-null cells reconstituted with caveolin-1. Scale bar $=20 \mu \mathrm{m}$. Data are representative of three independent experiments.

blasts were infected with an adenoviral vector containing wild-type caveolin-1 and wild-type PTEN. Cells infected with GFP were used as a control. An association of PTEN with caveolin-1 could be identified in the caveolin-1-null cells overexpressing both caveolin- 1 and PTEN but not in control caveolin-1-null cells infected with adenoviral-GFP (Figure 4C, left). Furthermore, when we reconstituted caveolin-1 in caveolin-1-null cells (but did not overexpress PTEN) we found an association of endogenous PTEN with caveolin-1 (Figure 4C, right), although the amount of PTEN associating with caveolin-1 was lower compared with caveolin-1-null cells overexpressing both PTEN and caveolin-1 (compare right and left panels). These data confirm that PTEN can associate with caveolin-1 in intact fibroblasts.

A

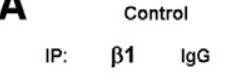

WB: $\beta 1$

WB: Cav-1
B

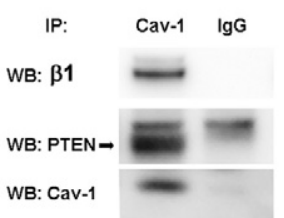

C

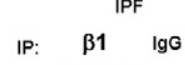

WB: $\beta 1$

WB: Cav-1
PTEN Colocalizes with Caveolin-1 at the Plasma Membrane

To assess whether caveolin-1 colocalizes with PTEN, we reconstituted caveolin-1-null fibroblasts with a wildtype caveolin-1 construct and analyzed PTEN and caveolin-1 staining by confocal microscopy. Caveolin1-null fibroblasts reconstituted with caveolin-1 showed a different pattern of staining compared with the null cells treated with empty vector. Reconstitution of caveolin-1null cells with caveolin-1 resulted in caveolin- 1 expression both within the plasma membrane and in a peri-nuclear distribution, and there was prominent PTEN expression in these cells (Figure 4D, left and middle). Importantly, immunofluorescence confocal microscopy delineated colocalization of PTEN and caveolin-1 at the plasma membrane in caveolin-1-null cells reconstituted with caveolin-1 (Figure 4D, right, arrows), but colocalization was not apparent in caveolin-1 nulls treated with empty vector (Figure 4E). Together with our biochemical studies, these results are consistent with the idea that caveolin-1 expression within the plasma membrane is a determinant of membrane-associated PTEN levels.

\section{A $\beta 1$ Integrin/Caveolin-1/PTEN Complex Is Present in Control Fibroblasts but Cannot be Detected in IPF Fibroblasts}

We have previously shown that the aberrant activation of the PI3K/Akt proliferation pathway attributable to inappro-
Figure 5. A $\beta 1$ integrin/caveolin-1/PTEN complex is present in control fibroblasts but cannot be detected in IPF fibroblasts. A: Control fibroblasts were cultured on polymerized collagen. $\beta 1$ integrin was immunoprecipitated lin-1 was immunoprecipitated and Western analysis for $\beta 1$ integrin, PTEN, or caveolin-1 was performed. Immunoprecipitation using IgG antibody is shown as a control. C: IPF fibroblasts were cultured on polymerized collagen. $\beta 1$ integrin was immunoprecipitated and Western analysis for caveolin-1 or $\beta 1$ integrin was performed. Note: Immunoprecipitation using IgG antibody is shown as a control. 
A
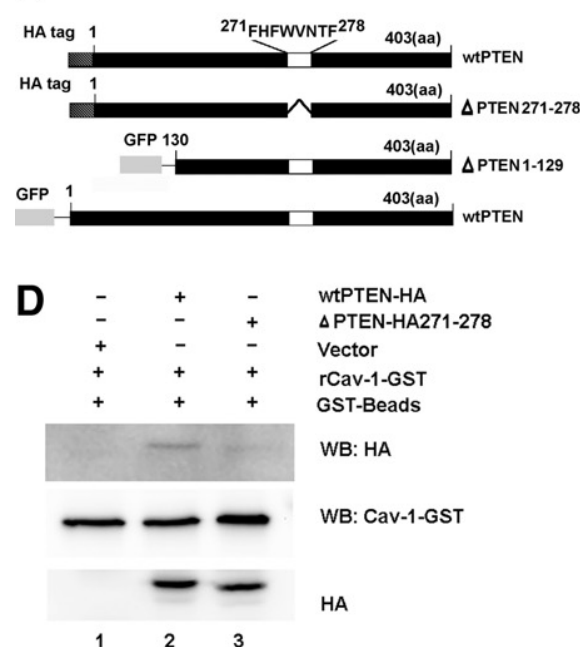

B

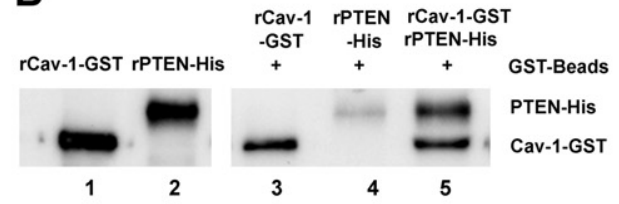

E

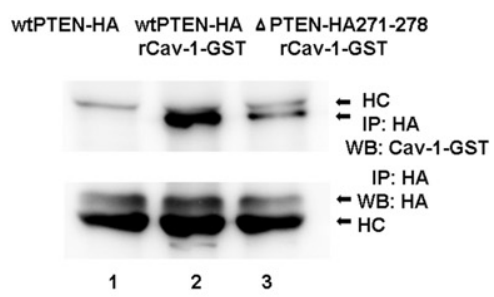

C

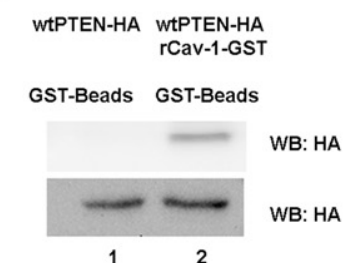

$\mathbf{F}$

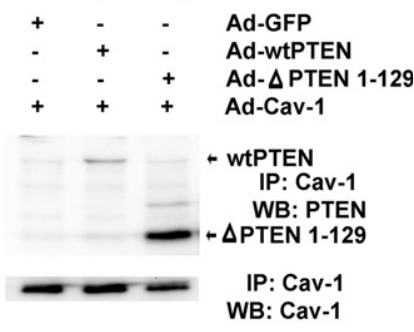

Figure 6. PTEN binds directly to caveolin-1. A: PTEN constructs showing: wild-type (wt) PTEN containing the 8-aa caveolin-1-binding motif; mutant PTEN construct absent the caveolin-1 binding motif ( $\triangle$ PTEN 271-278); and the $N$ terminus truncated PTEN construct absent the first 129 amino acids ( $\Delta$ PTEN 1-129). B: Cell free in vitro pull-down assay. Recombinant caveolin-1-GST (rCav-1-GST) was bound to GST beads and incubated with recombinant PTEN (rPTEN-His). PTEN incubated with GST beads without caveolin-1 was used as a control. Ln 1: rCav alone; Ln 2: rPTEN alone; Ln 3: rCav-1-GST incubated with GST beads alone; Ln 4: rPTEN incubated with GST beads alone; Ln 5 rPTEN incubated with rCav-1-GST and GST beads. C: Modified pull down assay. 293 cells were transfected with a HA-tagged wild-type PTEN construct. The cells were lysed and lysates incubated with: Ln 1: wild-type PTEN-HA and GST beads; Ln 2: wtPTEN-HA, rCav-1-GST, and GST beads. Shown is Western analysis of the GST beads using a HA tag antibody. Western analysis of whole cell lysates not exposed to beads using the HA tag antibody is shown as a loading control. D: Modified pull down assay. 293 cells were transfected with either HA-tagged wtPTEN, HA-tagged mutant PTEN construct where the 8-aa residues constituting the caveolin-1 binding sequence were deleted ( $\Delta$ PTEN 271-278), or empty vector. Cell lysates were incubated with rCav-1-GST and GST beads, and Western analysis of beads was performed using HA antibody and caveolin-1 antibody. Western analysis of whole cell lysates was performed as a loading control. Ln 1: empty vector; Ln 2: wtPTEN; Ln 3: $\Delta$ PTEN 271-278. E: 293 cells were transfected with either HA-tagged wtPTEN or HA-tagged mutant PTEN construct ( $\triangle$ PTEN 271-278). Cell lysates were incubated with rCav-1-GST and GST beads. PTEN was immunoprecipitated using a HA-tag antibody, and Western analysis of beads was performed using HA antibody and caveolin-1 antibody. Ln 1: wtPTEN (not incubated with rCav); Ln 2: wtPTEN + rCav-1-GST; Ln 3: $\triangle$ PTEN 271-278 + rCav-1-GST. HC denotes position of heavy chain. F: PTEN-null fibroblasts were infected with an adenoviral vector containing: Ln 1: empty vector (Ad-GFP) and wild-type caveolin-1 (Ad-cav-1); Ln 2: wild-type PTEN (Ad-wtPTEN) and wild-type caveolin-1 (Ad-cav-1); Ln 3: N-terminal truncated PTEN construct (Ad- $\triangle$ PTEN 1-129) and wild-type caveolin-1. Caveolin-1 was immunoprecipitated, and Western analysis for PTEN was performed. Shown as a loading control is immunoprecipitation of caveolin-1 followed by Western blotting for caveolin-1. Data are representative of three independent experiments.

priately low PTEN activity occurs during IPF fibroblast interaction with type I collagen via $\beta 1$ integrin. ${ }^{1}$ Prior studies suggest that caveolin-1 may associate with integrin and regulate integrin signaling. ${ }^{25-28}$ Since we have found that during control fibroblast interaction with collagen, PTEN associates with caveolin-1 and suppresses activation of Akt, we were interested in determining whether $\beta 1$ integrin also associates with caveolin-1 in a protein complex. We found that an endogenous protein complex consisting of caveolin-1, $\beta 1$ integrin, and PTEN could be coprecipitated from normal fibroblasts (Figure 5, $A$ and $B$ ). However, we were unable to detect a $\beta 1$ integrin/caveolin-1 protein complex during IPF fibroblast interaction with polymerized collagen (Figure $5 \mathrm{C}$ ). Of note, there was no significant difference in total $\beta 1$ integrin expression in IPF and control fibroblasts (data not shown). Together these data indicate that during normal fibroblast interaction with polymerized type I collagen, caveolin- 1 complexes with PTEN and $\beta 1$ integrin. This may place PTEN in close proximity to the integrin. However, during IPF fibroblast interaction with type I collagen, depleted caveolin-1 levels results in both an inability of $\beta 1$ integrin to associate with caveolin-1 and a reduction in PTEN association with caveolin-1. This may reduce the amount of PTEN in close association with $\beta 1$ integrin.

\section{PTEN Binds Directly to Caveolin-1}

Amino acid sequence analysis of PTEN indicates that PTEN contains the caveolin-1 consensus binding sequence $\Phi X \Phi X X X X \Phi$ corresponding to amino acids 271278 (FHFWVNTF) where $\Phi=$ aromatic amino acid phenylalanine (F) (Figure 6A). Because PTEN contains a caveolin-1 binding sequence, this suggested the possibility that PTEN directly interacts with caveolin-1. To analyze whether PTEN can directly interact with caveolin-1, we performed a cell free in vitro pull down assay where recombinant PTEN protein was incubated with recombinant GST-tagged caveolin-1 immobilized on GST beads. Considerable amounts of recombinant PTEN bound to caveolin-1 immobilized on the GST beads (Figure 6B, lane 5). Of note, a small amount of PTEN bound to the GST beads alone, indicating some nonspecific binding of recombinant PTEN to the beads (Figure 6B, lane 4). To verify this finding, we transfected 293 cells with a HAtagged wild-type PTEN construct. The cells were lysed and lysates incubated with recombinant caveolin-1-GST and GST beads or with GST beads alone as a control. We found that PTEN (HA tagged) bound to the recombinant caveolin-1 whereas no PTEN could be detected when the cell lysates were incubated with GST beads alone (Figure 
$6 C)$. These data strongly suggest that PTEN is capable of binding directly to caveolin-1.

To assess whether PTEN binds to caveolin-1 via its caveolin-1 binding sequence, we generated a HAtagged mutant PTEN construct in which the 8-aa caveolin-1 binding sequence was deleted. We then examined the ability of the mutated PTEN construct to bind to caveolin-1. We first overexpressed either HA-tagged wild-type PTEN or the HA-tagged mutant PTEN ( $\triangle$ PTEN 271-278) construct in 293 cells and performed pull-down assays where the cell lysates were incubated with recombinant caveolin-1 GST and GST beads. We found that wild-type PTEN (HA tagged) bound to caveolin-1 (Figure $6 D$, lane 2), whereas the $\triangle P T E N$ 271-278 construct absent the caveolin-1 binding domain did not (Figure 6D, lane 3). We also performed a complementary experiment where we overexpressed HA-tagged wild-type PTEN or the HA-tagged mutant PTEN construct in 293 cells, and the cell lysates were incubated with caveolin-1-GST and GST beads and the lysates immunoprecipitated with HAtagged antibody. A large quantity of HA-tagged wild-type PTEN bound to caveolin-1 (Figure 6E, lane 2), whereas only a small amount of HA-tagged mutant PTEN associated with recombinant caveolin-1 (Figure 6E, lane 3). In addition, we examined the ability of a truncated PTEN construct to associate with caveolin-1. In this truncated PTEN construct, the first 129 amino acids of the PTEN amino terminus have been removed but the caveolin-1 binding motif remains intact. Both wild-type PTEN (Figure 6F, Lane 2) and truncated PTEN containing the caveolin1-binding motif (Figure 6F, Lane 3) immunoprecipitated with caveolin-1. Together, these data suggest that PTEN uses the caveolin-1-binding domain to directly associate with caveolin-1. However, our data do not rule out the possibility that other domains of PTEN (e.g., within the C terminus) may also contribute in its ability to bind to caveolin-1.

\section{The Absence of Caveolin-1 Correlates with Diminished PTEN Activity and Enhanced Akt Activity}

PTEN is a major inhibitor of the integrin/PI3K/Akt signal pathway. Because membrane-associated PTEN levels are decreased in caveolin-1-null cells, we sought to determine the effect of reconstitution of caveolin-1 in caveolin-1-null cells on PTEN and Akt activity. PTEN activity in cells reconstituted with caveolin-1 was increased by $\sim 59 \%$ compared with caveolin-1-null cells reconstituted with GFP only (Figure 7A). Consistent with the increase in PTEN activity, we found that phospho-Akt levels were decreased following reconstitution of caveolin-1 in caveolin1-null cells compared with cells treated with empty vector (Figure 7B). These data support the idea that the level of caveolin-1 is a determinant of membrane-associated PTEN levels and activity. Low caveolin-1 expression leads to diminished membrane PTEN levels and low PTEN activity which augments Akt activity.
A

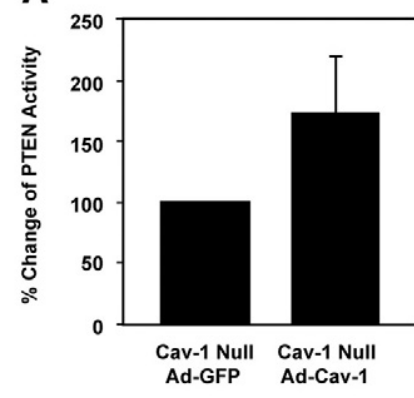

B



D

Cav-1 Null Ad-GFP Ad-DN-Akt

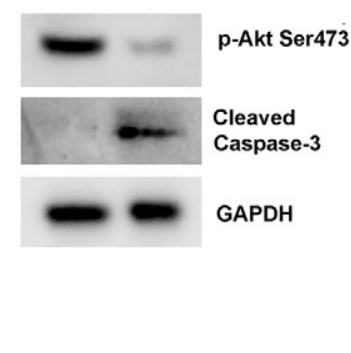

E

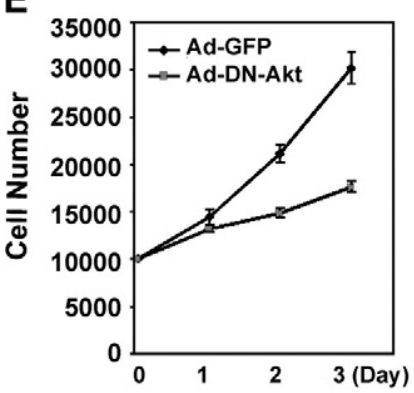

$\mathbf{F}$



Figure 7. The absence of caveolin-1 diminishes PTEN activity and enhances Akt activity while promoting exaggerated proliferation and resistance to apoptosis. Caveolin-1-null cells were infected with an adenoviral vector containing wither wild-type caveolin-1 (Cav-1 Null Ad-GFP) or empty vector (Cav-1 Null Ad-Cav-1). PTEN activity (A) and the level of phosphorylated Akt (B) were assessed 48 hours after infection. C: Caveolin-1-null fibroblasts and wild-type fibroblasts were plated on either tissue culture plates (TC) or polymerized collagen (PC) in growth factor-replete media and cell number determined as a function of time. D: Caveolin-1-null fibroblasts were infected with an adenoviral vector containing dominant negative Akt or empty virus and cultured in the presence of media containing $10 \%$ serum. The levels of phospho-Akt and cleaved caspase 3 were examined by Western analysis. $\mathbf{E}$ and $\mathbf{F}$ : The proliferation of caveolin-1-null cells expressing DN-Akt or GFP-control was quantified by assessing cell number at 24, 48, and 72 hours postinfection (E) or by BrdU uptake at 72 hours (F). Error bars represent SEM. Data are representative of three independent experiments.

Caveolin-1-Null Fibroblasts Display Exaggerated Proliferation and Resistance to Apoptosis Compared with Caveolin-1 Wild-Type Cells Cultured on Polymerized Type I Collagen Matrices

The PI3K/Akt pathway regulates cell proliferation and survival. Because our data indicate that caveolin-1-null fibroblasts manifest depleted membrane PTEN expression/activity and enhanced Akt activity, we next examined the effect of depletion of caveolin-1 on fibroblast proliferation and viability. We have previously demon- 
strated that polymerized type I collagen suppresses normal fibroblast proliferation by a mechanism involving maintenance of high PTEN activity. Thus, we sought to investigate whether polymerized collagen exerted proliferation-suppressive properties on caveolin-1-null fibroblasts. To address this issue, we cultured caveolin-1 wild-type and null fibroblasts on either tissue culture dishes without matrix or on polymerized collagen matrices for 4 days in the presence of $10 \%$ serum. We did not find a major difference in the proliferation of caveolin-1null and wild-type fibroblasts on tissue culture plates. Moreover, similar to normal human lung fibroblasts, polymerized collagen potently suppressed the proliferation of caveolin-1 wild-type fibroblasts. However, we found that the degree of suppression of cell proliferation by polymerized collagen was less prominent in caveolin-1null cells, a finding similar to our published results for IPF fibroblasts. ${ }^{1}$ Caveolin-1-null fibroblast cell numbers were $80 \%$ higher than caveolin-1 wild-type fibroblasts cultured on polymerized collagen for 4 days (Figure $7 \mathrm{C}$ ). This suggests that depletion of caveolin-1 levels augments Akt activity and confers fibroblasts with an increased ability to elude the proliferative-suppressive properties of polymerized collagen. To verify this, we knocked down Akt by using a dominant-negative Akt construct as we have described and examined caveolin-1-null cell proliferation. ${ }^{1}$ Dominant negative Akt reduced the level of phospho-Akt (Figure 7D) and suppressed the ability of caveolin-1-null cells to proliferate (Figure 7, E and F). Reduction in the level of phospho-Akt may also promote apoptosis. Using FACS analysis to quantify the level of apoptosis, we observed an increase in apoptosis in caveolin-1-null cell cultures at 48 hours after knock-down of Akt compared with control (19\% versus $8 \%$ ). Consistent with this, we also detected the presence of cleaved caspase 3 in caveolin-1-null cells treated with dominant negative Akt (Figure 7D). This indicates that in caveolin1-null cells, knock-down of Akt promotes a low level of apoptosis in addition to suppressing proliferation. Furthermore, we noted that when caveolin-1 wild-type cells were cultured on polymerized collagen their cell numbers declined. Therefore, we also examined the level of apoptosis in caveolin-1 wild-type and null fibroblasts cultured on polymerized collagen for 3 days. We found that the level of apoptosis was higher in caveolin-1 wild-type fibroblasts cultured on polymerized collagen compared with caveolin-1-null cells (17\% versus $2 \%$; see supplemental Figure 3 at $h$ ttp://ajp.amjpathol.org). These data suggest that an absence of caveolin-1 may confer resistance to apoptosis in response to culture on polymerized type I collagen.

\section{Knock-Down of Caveolin-1 in Control Fibroblasts Reduces Membrane-Associated PTEN Levels, Augments Phospho-Akt Levels, and Increases Proliferation}

We next sought to assess whether knock-down of caveolin-1 levels in control fibroblasts will confer an IPF fibro-



Figure 8. Knock-down of caveolin-1 in control fibroblasts reduces membrane PTEN levels, augments phospho-Akt levels, and increases proliferation. Control fibroblasts were transfected with caveolin-1 or control siRNA, or not treated (NT). Caveolin-1, membrane PTEN, phospho-Akt, and GAPDH expression were examined by Western analysis. Data are representative of three independent experiments.

blast-like phenotype. To do this, we knocked down caveolin-1 expression in control lung fibroblasts using caveolin-1 siRNA and examined the effect on membraneassociated PTEN levels, Akt activity, and cell growth. We found that knock-down of caveolin-1 in control fibroblasts modestly decreased caveolin-1 expression as well as membrane-associated PTEN levels and that knock-down of caveolin-1 augmented the level of phospho-Akt (Figure 8). We next assessed the effect of knock-down of caveolin-1 on the proliferation of control fibroblasts plated on polymerized type I collagen in the presence of serum for 48 hours. The percentage of cells staining positive for $\mathrm{BrdU}$ was roughly two-fold higher in control fibroblasts in which caveolin-1 had been knocked-down compared with cells treated with control siRNA where elevated levels of caveolin-1 and membrane-associated PTEN were maintained (35.1\% versus $14.7 \%$; see supplemental Figure 4 at $h t t p: / / a j p . a m j p a t h o l . o r g)$. Our data indicate that low caveolin-1 levels correlate with augmented fibroblast proliferation and suggest that depletion of caveolin-1 confers control fibroblasts with the ability to circumvent the anti-proliferative properties of polymerized collagen.

\section{Overexpression of Caveolin-1 in IPF Fibroblasts Augments Membrane-Associated PTEN Levels and Suppresses Akt Activity}

Our studies using control fibroblasts indicate that caveolin-1 expression is a determinant of PTEN expression and activity. To examine whether caveolin-1 levels are a determinant of membrane-associated PTEN expression in IPF fibroblasts, we infected IPF fibroblasts with an adenoviral vector containing a wild-type caveolin-1 construct and analyzed PTEN expression. Fibroblasts infected with empty vector served as control. IPF fibroblasts infected with the adenoviral vector containing the caveolin-1 construct displayed marked up-regulation of caveolin-1 protein expression and this correlated with increased membrane-associated PTEN levels (Figure 9A). To determine the effect of this increase in membrane-associated PTEN on Akt activity, we analyzed Akt phosphorylation. The level of phosphorylated serine 473 of Akt was modestly suppressed in IPF fibroblasts overexpressing caveolin-1 compared with IPF fibroblasts infected with empty vector (Figure 9A). Interestingly, the level of cyclin D was markedly suppressed in IPF fibroblasts overexpressing caveolin-1 compared with control. Together, these data 
B

A

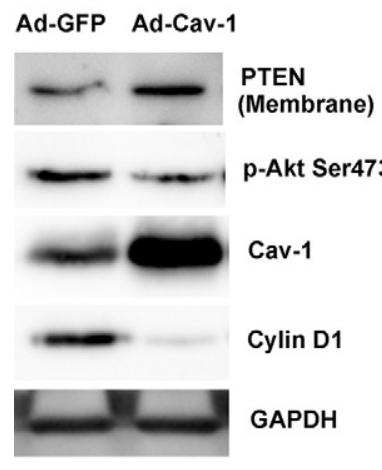

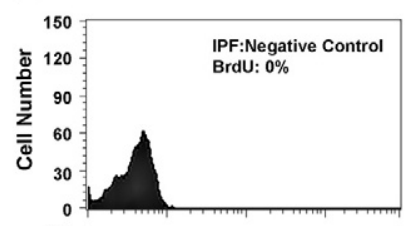

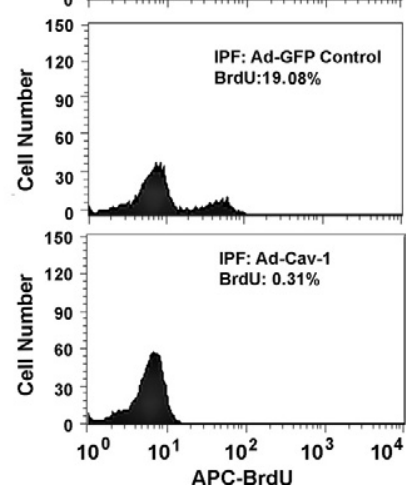

Figure 9. Overexpression of caveolin-1 in IPF fibroblasts augments membrane PTEN levels and suppresses Akt activity. IPF fibroblasts were infected with an adenovirus containing wild-type caveolin-1 (Ad-wtCav1) or empty vector (Ad-GFP) and cultured on tissue culture dishes in the presence of serum for 48 hours. A: Shown is Western analysis of caveolin-1, membrane PTEN, phosphorylated Akt, and cyclin D levels. GAPDH is used as a loading control. B: IPF fibroblasts were infected with an adenoviral vector containing wild-type caveolin-1 (Ad-Cav-1) or empty vector (Ad-GFP), and proliferation was quantified by BrdU uptake by FACS. Negative control = cells stained with secondary antibody only. Data are representative of three independent experiments.

strongly suggest that caveolin-1 expression is a determinant of membrane PTEN expression and that low caveolin-1 expression is responsible for the inappropriately low PTEN activity and the pathologically activated PI3K/Akt pathway that is characteristic of IPF fibroblasts.

We also examined the effect of overexpression of caveolin-1 on IPF fibroblast proliferation and viability. The cells were infected with an adenoviral vector containing either wild-type caveolin-1 or empty vector and cultured on tissue culture dishes. The level of BrdU uptake in IPF fibroblasts infected with empty vector was 19\%. In contrast, overexpression of caveolin-1 nearly completely suppressed BrdU uptake (Figure 9B), indicating that overexpression of caveolin-1 effectively suppresses IPF fibroblast proliferation. We also analyzed the effect of overexpression of caveolin-1 on IPF fibroblast viability. Somewhat surprisingly, we found that the level of apoptosis of IPF fibroblasts overexpressing caveolin-1 was significantly higher than empty vector control $(\sim 31 \%$ versus


org). These data suggest that the pathologically low level of caveolin-1 in IPF fibroblasts may confer IPF fibroblasts with the ability to elude the proliferation-suppressive effects of polymerized type I collagen and an apoptoticresistant phenotype.

\section{Discussion}

Idiopathic pulmonary fibrosis is a prototypical fibroproliferative disorder. It is characterized by progressive replacement of alveolar airspaces with proliferating fibro-

blasts and their major connective tissue product-type I collagen. ${ }^{29}$ However, this observation-that IPF is characterized by the relentless proliferation of fibroblasts in a matrix of polymerized type I collagen-was at odds with the long-known fact that polymerized type I collagen is a potent suppressor of fibroblast proliferation. Focusing on this disparate observation, we have found that IPF fibroblasts have eluded the proliferative-suppressive effects of polymerized type I collagen. We have demonstrated that the mechanism involves aberrant activation of the PI3K/Akt pathway due to inappropriately low activity of the tumor suppressor phosphatase PTEN. ${ }^{1}$ We have found that PTEN activity is low because of decreased levels of PTEN at the plasma membrane-the location where PTEN inhibits phosphatidylinositol-3,4,5-triphosphate. ${ }^{1}$ Interestingly, a prior study has found that IPF is also characterized by low levels of the integral membrane protein caveolin-1. ${ }^{21}$ Here we show that reduced expression of caveolin- 1 is responsible for the decreased level of membrane-associated PTEN and the pathological activation of the PI3K/Akt signal in IPF fibroblasts. We demonstrate that the level of caveolin-1 is a major determinant of membrane-associated PTEN expression.

Caveolin-1 exists in high-molecular-mass oligomers of $350 \mathrm{kDa}$ and can be found in mesh-like structures juxtaposed to the inner plasma membrane..$^{30-32}$ Caveolin-1's scaffolding domain (residues 82-101) anchors caveolin-1 to the membrane. ${ }^{33}$ Caveolin-1 has been linked to the regulation of membrane-associated signal transduction pathways that control key cellular functions including proliferation. ${ }^{18,34-38}$ In this study, our immunofluorescence and biochemical experiments demonstrate that plasma membrane caveolin-1 expression is depleted in primary lung fibroblasts derived from patients with IPF and that overexpression of caveolin-1 in IPF fibroblasts results in a prominent peri-nuclear localization of caveolin-1 and restoration of caveolin-1 expression at the plasma membrane. Our study links depletion of caveolin-1 expression in fibroblasts with a pathological fibroblast phenotype characterized by an exaggerated ability to proliferate that may contribute to aberrant tissue repair

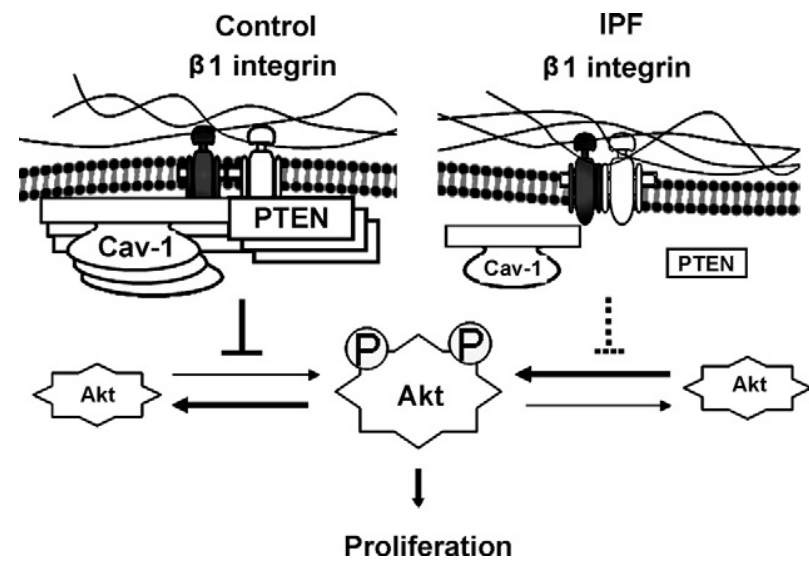

Figure 10. Working model of IPF fibroblast phenotype. Schematic illustrating the molecular mechanism underlying the ability of IPF fibroblasts to elude the proliferation-suppressive properties of polymerized type I collagen (see text for details). 
and fibrosis. Consistent with this, recent studies suggest that a loss of caveolin-1 expression may be a marker of the cancer-associated fibroblast phenotype, further linking depletion of caveolin-1 with a pathological fibroblast phenotype. ${ }^{37,38}$

We have previously demonstrated that membrane-associated PTEN levels and activity are low in IPF fibroblasts. ${ }^{1}$ This results in pathological activation of the PI3K/ Akt signal pathway and confers IPF fibroblasts with the ability to elude the antiproliferative properties of polymerized type I collagen. We have also previously demonstrated that PTEN-deficient mice display increased lung fibrosis in response to bleomycin-induced lung injury caused by a more durable fibroproliferative response, confirming the importance of the PTEN/Akt axis in regulating the degree of fibroproliferation and subsequent fibrosis. ${ }^{1}$ Considerable experimental evidence indicates that PTEN localizes to the plasma membrane where it is in the correct location to inhibit the PI3K/Akt signal. ${ }^{9,14-16}$ Here we provide substantial evidence that the depletion of caveolin-1 at the membrane is responsible for low membrane-associated PTEN expression and activation of Akt in IPF fibroblasts, which facilitates their pathological proliferation. First, using caveolin-1-null cells treated with an adenoviral vector containing wild-type caveolin-1 we demonstrate that reconstitution of caveolin-1 results in the colocalization of caveolin-1 and PTEN at the plasma membrane. We show that this augments PTEN activity while suppressing Akt activity. Second, we show that caveolin-1-null cells display exaggerated proliferation and importantly, we demonstrate that down-regulation of Akt function in caveolin-1-null cells suppresses their ability to proliferate. Third, we demonstrate that knock-down of caveolin-1 in control fibroblasts reduces membraneassociated PTEN levels while increasing phospho-Akt levels and their ability to proliferate. Fourth and most importantly, we demonstrate that restoration of caveolin-1 levels in IPF fibroblasts repletes membrane-associated PTEN levels, thereby diminishing Akt activity and suppressing IPF fibroblast proliferation. Finally, our immunofluorescent confocal microscopic analysis of IPF lung tissue demonstrates that $\alpha$ smooth muscle actin expressing myofibroblasts within the fibroblastic focus, in a zone adjacent to the overlying epithelium, display low-intensity expression of both caveolin-1 and PTEN, thus confirming correlation of caveolin-1 and PTEN expression in myofibroblasts within the fibroblastic focus.

Amino acid analysis of PTEN indicates that PTEN contains a caveolin-1 consensus binding sequence. We provide evidence that PTEN directly interacts with caveolin-1. Our cell-free pull-down assay using recombinant caveolin-1 and wild-type PTEN protein suggest strong avidity between caveolin-1 and PTEN, whereas we found reduced PTEN association with caveolin-1 when we used our mutant PTEN construct in which the caveolin-1-binding domain had been deleted. Furthermore, our co-immunoprecipitation experiments demonstrate that in control fibroblasts, where endogenous caveolin- 1 levels are high, caveolin- 1 can associate with both PTEN and $\beta 1$ integrin in a protein complex. In contrast, in IPF fibroblasts, which display very low levels of endogenous caveolin-1 and membrane-associated PTEN levels, we were unable to detect PTEN in a complex with caveolin-1. Only when PTEN and caveolin-1 were overexpressed in IPF fibroblasts were we able to detect the proteins in a complex. In addition, in IPF fibroblasts, $\beta 1$ integrin could not be detected in a complex with caveolin-1. On the basis of our studies we suggest a model where during normal fibroblast interaction with polymerized collagen via $\beta 1$ integrin, PTEN accumulates at the plasma membrane by association with the meshlike caveolin-1 structure in a protein complex also containing $\beta 1$ integrin (Figure 10). This positions PTEN in an optimal location to inhibit phosphoinositol 3,4,5 triphosphate generated by integrin-matrix interaction. The accumulation of PTEN at the membrane results in a membrane microenvironment characterized by high phosphatase activity, which represses the integrin/PI3K/Akt signal. However, we suggest that in IPF fibroblasts, depletion of membrane-associated caveolin-1 results in reduced accumulation of PTEN at the membrane in a complex with caveolin-1. In addition, because of depleted membrane caveolin- 1 levels, there is reduced $\beta 1$ integrin association with caveolin-1. Together, this limits the accessibility of PTEN to phosphoinositol 3,4,5 triphosphate generated by $\beta 1$ integrin-matrix interaction, thereby facilitating the aberrant activation of Akt.

In conclusion, our studies demonstrate that in IPF fibroblasts, a lack of caveolin-1 expression in the plasma membrane reduces membrane-associated PTEN levels and activity. This creates a membrane microenvironment depleted of inhibitory phosphatase activity and favorable for the pathological activation of the PI3K/Akt signal pathway. This confers IPF fibroblasts with a phenotype characterized by the ability to circumvent the proliferationsuppressive properties of polymerized type I collagen.

\section{Acknowledgments}

We thank Augustine Choi for the gift of the caveolin-1-null and wild-type fibroblasts and Eric White for the PTEN-null and wild-type fibroblasts.

\section{References}

1. Xia H, Diebold D, Nho R, Perlman D, Kahm J, Kleidon J, Avdulov A, Peterson M, Bitterman PB, Henke CA: Pathological integrin signaling enhances proliferation of primary lung fibroblasts from patients with idiopathic pulmonary fibrosis. J Exp Med 2008, 205:1659-1672

2. Schor SL: Cell migration and proliferation on collagen substrata in vitro. J Cell Sci 1980, 41:159-175

3. Rhudy RW, McPherson JM: Influence of the extracellular matrix on the proliferative response of human skin fibroblasts to serum and purified platelet-derived growth factor. J Cell Physiol 1988, 137:185-191

4. Koyama H, Raines EW, Bornfedlt KE, Roberts JM, Ross R: Fibrillar collagen inhibits arterial smooth muscle proliferation through regulation of cdk2 inhibitors. Cell 1996, 87:1069-1078

5. White ES, Atrasz RG, Hu B, Phan SH: Stambolic V, Mak TW, Hogaboam $\mathrm{CM}$, Flaherty KR, Martinez FJ, Kontos CD, Toews GB: negative regulation of myofibroblast differentiation by phosphatase and tensin homologue deleted on chromosome ten. Am J Respir Crit Care Med 2006, 173:112-121

6. Yamada KM, Araki M: Tumor suppressor PTEN: modulator of cell signaling, growth, migration and apoptosis. J Cell Science 2002 , 114:2375-2382 
7. Georgescu MM, Kirsch KH, Akagi T, Shishido T, Hanafusa H: The tumor-suppressor activity of PTEN is regulated by its carboxyl-terminal region. Proc Natl Acad Sci U S A 1999, 96:10182-10187

8. Stambolic V, Tsao MS, Macpherson D, Suzuki A, Chapman WB, Mak TW: High incidence of breast and endometrial neoplasia resembling human Cowden Syndrome in pten+/- mice. Cancer Res 2000, 60:3605-3611

9. Lee JO, Yang H, Georgescu MM, Cristofano AD, Maehama T, Shi Y, Dixon JE, Pandolfi P, Pavletich NP: Crystal structure of the PTEN tumor suppressor: implications for its phosphoinositide phosphatase activity and membrane association. Cell 1999, 99:323-334

10. Tamura M, Gu J, Danen EHJ, Takino T, Miyamoto S, Yamada KM: PTEN interactions with focal adhesion kinase and suppression of the extracellular matrix-dependent phosphatidylinositol 3-kinase/Akt cell survival pathway. J Biol Chem 1999, 274:20693-20703

11. Stambolic V, Suzuki A, de la Pompa JL, Brothers GM, Mirtsos C, Sasaki T, Ruland J, Penninger JM, Siderovski DP, Mak TW: Negative regulation of PKB/Akt dependent cell survival by the tumor suppressor PTEN. Cell 1998, 95:29-39

12. Lu Y, Yu Q, Liu JH, Zhang J, Wang H, Koul D, McMurray JS, Fang X, Yung WKA, Siminovitch KA, Mills GB: Src family protein-tyrosine kinases alter the function of PTEN to regulate phosphatidyinositol 3-kinase/Akt cascades. J Biol Chem 2003, 278:40057-40066

13. Li DM, Sun H: TEP1, encoded by a candidate tumor suppressor locus, is a novel protein tyrosine phosphatase regulated by transforming growth factor $\beta$. Cancer Res 1997, 57:2124-2129

14. Torres J, Pulido R: The tumor suppressor PTEN is phosphorylated by the protein kinase CK2 at its C terminus. J Biol Chem 2001, 276: 993-998

15. Vazquez F, Ramaswamy S, Nakamura N, Sellers WR: Phosphorylation of the PTEN tail regulates protein stability and function. Mol Cell Biol 2000, 20:5010-5018

16. Wu X, Hepner K, Castelino-Prabhu, Do D, Kaye MB, Yuan XJ, Wood $J$, Ross C, Sawyers CL, Whang YE: Evidence for regulation of the PTEN tumor suppressor by a membrane-localized multi-PDZ domain containing scaffold protein MAGI-2. Proc Natl Acad Sci U S A 2000, 97:4233-4238

17. Shi F, Sottile J: Caveolin-1-dependent beta1 integrin endocytosis is a critical regulator of fibronectin turnover. J Cell Sci 2008, 121:23602371

18. Li L, Ren CH, Tahir SA, Ren C, Thompson TC: Caveolin-1 maintains activated Akt in prostate cancer cells through scaffolding domain binding site interactions with and inhibition of serine/threonine protein phosphatases PP1 and PP2A. Mol Cell Biol 2003, 23:9389-9404

19. Drab M, Verkade P, Elger M, Kasper M, Lohn M, Lauterbach B, Menne J, Lindschau C, Mende F, Luft FC, Schedl A, Haller H, Kurzchalia TV: Loss of caveolae, vascular dysfunction, and pulmonary defects in caveolin-1 gene-disrupted mice. Science 2001, 293: 2449-2452

20. Galdo FD, Sotgia F, de Almeida CJ, Jasmin JF, Musick M: Lisanti MP. Jimenez SA: decreased expression of caveolin-1 in patients with systemic sclerosis: crucial role in the pathogenesis of tissue fibrosis Arthritis Rheum 2008, 58:2854-2865

21. Wang XM, Zhang Y, Kim HP, Zhou Z, Feghali-Bostwick CA, Lui F, Ifedigbo E, Xu X, Oury TD, Kaminiski N, Choi AM: Caveolin-1: a critical regulator of lung fibrosis in idiopathic pulmonary fibrosis. J Exp Med 2006, 203:2895-2906

22. Couet J, Li S, Okamoto T, Ikezu T, Lisanti MP: Identification of peptide and protein ligands for the caveolin-scaffolding domain. J Biol Chem 1997, 272:6525-6533

23. American Thoracic Society. American Thoracic Society/European Respiratory Society International Multidisciplinary Consensus Classification of the Idiopathic Interstitial Pneumonias. Am J Respir Crit Care Med 2002, 165:277-304
24. Chen B, Polunovsky V, White J, Blazar B, Nakleh R, Jessurun J, Peterson M, Bitterman P: Mesenchymal cells isolated after acute lung injury manifest an enhanced proliferative phenotype. J Clin Invest 1992, 90:1778-1785

25. Chapman HA, Wei Y, Simon DI, Waltz DA: Role of urokinase receptor and caveolin in regulation of integrin signaling. Thromb Haemost 1999, 82:291-297

26. Schwab W, Gavlik JM, Beichler T, Funk RH, Albrecht S, Magdolen V, Luther T, Kasper M, Shakibaei M: Expression of the urokinase-type plasminogen activator receptor in human articular chondrocytes: association with caveolin and beta 1-integrin. Histochem Cell Biol 2001, 115:317-323

27. Martin S, Cosset EC, Terrand J, Maglott A, Takeda K, Dontenwill M: Caveolin-1 regulates glioblastoma aggressiveness through control of alpha 5 beta 1 integrin expression and modulates glioblastoma responsiveness to SJ749, an alpha 5 beta 1 integrin antagonist. Biochim Biophys Acta 2009, 1793:354-367

28. Upla P, Marjomaki V, Kankaanpaa P, Ivaska J, Hyypia T, Van Der Goot FG, Heino J: Clustering induces a lateral redistribution of alpha 2 beta 1 integrin from membrane rafts to caveolae and subsequent protein kinase C-dependent internalization. Mol Cell Biol 2004, 15:625-636

29. Kuhn C, McDonald JA: The roles of the myofibroblast in idiopathic pulmonary fibrosis. Am J Pathol 1991, 138:1257-1265

30. Spisni E, Tomasi V, Cestaro A, Tosatta SCE: Structural insights into the function of human caveolin 1. Biochem Biophys Res Comm 2005, 338: 1383-1390

31. Sargiacomo M, Scherer PE, Tang Z, Kubler E, Song KS, Sanders MC Lisanti MP: Oligermeric structure of caveolin: implications for caveolae membrane organization. Proc Natl Acad, Sci U S A 1995, 92 : 9407-9411

32. Nishida T, Arii T, Takaoka A, Yoshimura R, Endo Y: Three-dimensional, computer-tomographic analysis of membrane protein (TrkA, caveolin, clathrin) in PC12 cells. Acta Histochem Cytochem 2006, 40:93-99

33. Schegel A, Schwab RB, Scherer PE, Lisanti MP: A role for the caveolin scaffolding domain in mediating the membrane attachment of caveolin-1. The caveolin scaffolding domain is both necessary and sufficient for membrane binding in vitro. J Biol Chem 1999, 274: 22660-22667

34. Cohen AW, Combs TP, Scherer PE, Lisanti MP: Role of caveolin and caveolae in insulin signaling and diabetes. Am J Physiol Endocrinol Metab 2003, 285:E1151-E1160

35. Mineo C, James GL, Smart EJ, Anderson RG: Localization of epidermal growth factor-stimulated Ras/Raf-1 interaction to caveolae membrane. J Biol Chem 1996, 271:11930-11935

36. Liu P, Ying Y, Anerson RG: Platelet-derived growth factor activates mitogen-activated protein kinase in isolated caveolae. Proc Natl Acad Sci U S A 1997, 94:13666-13670

37. Sotgia F, Del Galdo F, Casimiro MC, Bonuccelli G, Mercier I, WhitakerMenezes D, Daumer KM, Zhou J, Wang C, Katiyar S, Xu H, Bosco E, Quong AA, Aronow B, Witkiewicz Ak, Minetti C, Frank PG, Jimenez SA, Knudsen ES, Pestell RG, Lisanti MP: Caveolin-1 -/- null mammary stromal fibroblasts share characteristics with human breast cancer-associated fibroblasts. Am J Pathol 2009, 174:746-761

38. Mercier I, Casimiro MC, Wang C, Rosenberg AL, Quong J, Minkeu A Allen KG, Danilo C, Sotgia F, Bonuccelli G, Jasmin JF, Xu H, Bosco E, Aronow B, Witkiewicz A, Pestell RG, Knudsen ES, Lisanti MP: Human breast cancer-associated fibroblasts (CAFs) show caveolin-1 downregulation and RB tumor suppressor functional inactivation: implications for the response to hormonal therapy. Cancer Biol Ther 2008, 7:1212-1225 